\title{
Traffic Modeling of Wireless Body Area Network
}

\author{
Humaid S. Alshamsi ${ }^{1}$, Hessa S. Al-Shamisi ${ }^{2}$, Hongying Mengi ${ }^{3}$ \\ ${ }^{I}$ Dept. Electronic and Computer Engineering Florida Institute of Technology Melbourne FL, USA \\ ${ }^{2}$ College of Information Technology United Arab Emirates University Al-Ain, UAE \\ ${ }^{3}$ Dept. Electronic and Computer Engineering Brunel University London UB8 3PH, UK
}

\begin{abstract}
Wireless Body Area Network (WBAN) is required to provide quality of service (QoS) guarantees for transporting and processing life threatening gathered data. One important aspect of providing QoS guarantees understands the traffic dynamics of the WBAN. Towards this end, the objective of this project is to study the traffic dynamics of WBAN and attempt to equip a traffic model of WBAN over ZigBee. In this project, we implemented three types of network topologies to study traffic dynamics. We studied the end-to-end delay of traffic, the sent traffic histogram and the received traffic histogram. We concluded that the current OPNET ZigBee simulator is not sufficient to provide complete and solid results for traffic modeling. Additionally, we observed that the star topology incurs the lowest end-to-end delay and the tree topology incurs the highest.
\end{abstract}

Key Words: WBAN, traffic modeling, QoS, end-to-end delay.

\section{Introduction}

Wireless Body Area Network is a special type of Wireless Personal Area Network. WBAN is normally used for remotely monitoring and reporting of users' health vitals. Composed of various sensors that are either wearable or implanted, a WBAN relies on wireless connectivity to transfer the collected data to an Internetbased data management service. A typical remote monitoring system comprises a fitted WBAN, a gateway between the WBAN and the Internet, a service for data transfer over the Internet; and a service for data processing, providing interface and alerts to the caregivers. WBANs have great potential for several applications including remote medical diagnosis, interactive gaming, and military applications [1].

WBAN employs different types of physical layer technologies, which results in a wide range of data rates and latency based on the technology employed. In the following we summarize the most used technologies in WBAN: Bluetooth is a short-range radio transmission system that is useful for sending data between electronic devices Bluetooth supports voice and data. Impulse radio (IR)/Ultra Wide Band (UWB) technology is low cost and uses low power for different types of communications The main reason is that this technology uses short pulses ( 0.1 to 1.5 Nanoseconds) and very low average power in the milliwatt range. ZigBee technology is a wireless controlled and sensor networking with QoS support. Also, it is low cost, requires low power and provides high level communication. This technology is used in scientific and ISM medical radio bands. The table 1, provides a study of the popular personal wireless communication standards, evaluating their main features and behaviors in terms of various metrics, including the transmission time, data coding efficiency, complexity, and power channel access and modulations.

Table 1: Summary of characteristics of UWB, 60 GHZ WPAN, and ZigBee

\begin{tabular}{|c|c|c|c|}
\hline Systems & UWB & $60 \mathrm{GHz}$ WPAN & ZigBee \\
\hline Standard status & Dissolved in IEEE & In progress & Approved \\
\hline Frequency allocation & $3.1-10.6 \mathrm{GHz}$ & $\begin{array}{l}57-64 \mathrm{GHz} \text { (U.S.) } \\
59-66 \mathrm{GHz} \text { (Japan) } \\
57-66 \mathrm{GHz} \text { (Europe) }\end{array}$ & $\begin{array}{l}2.4-2.4835 \mathrm{GHz}^{\prime} \\
901-928 \mathrm{MHz}^{2} \\
868-868.6 \mathrm{MHz}^{4}\end{array}$ \\
\hline Channel bandwidth & $\geq 500 \mathrm{MHz}$ & Not yet available & $2,10.6,20.3 \mathrm{MH}$ \\
\hline $\begin{array}{l}\text { Number of RF chan- } \\
\text { nels }\end{array}$ & $\begin{array}{l}2^{5} \\
14^{6}\end{array}$ & 4 (IEEE $802.15 .3 \mathrm{c}$ ) & $16,{ }^{1} 10,21^{4}$ \\
\hline Maximum data rate & $\begin{array}{l}100 \mathrm{Mb} / \mathrm{s}(10 \mathrm{~m}) \\
200 \mathrm{Mb} / \mathrm{s}(4 \mathrm{~m}) \\
480 \mathrm{Mb} / \mathrm{s} \text { (optional) }\end{array}$ & $\begin{array}{l}2 \mathrm{~Gb} / \mathrm{s} \text { (at least) } \\
\geq 3 \mathrm{~Gb} / \mathrm{s} \text { (optional) }\end{array}$ & $\begin{array}{l}250 \mathrm{~kb} / \mathrm{s}^{1} \\
40 \mathrm{~kb} / \mathrm{s}^{2} \\
20 \mathrm{~kb} / \mathrm{s}^{4}\end{array}$ \\
\hline Modulation & DSSS, 5 OFDM 6 & Not yet available & BPSK, 2,4 OQPSK1 \\
\hline Maximum coverage & $\sim 10 \mathrm{~m}$ & $-20 \mathrm{~m}$ & $\sim 20 \mathrm{~m}$ \\
\hline
\end{tabular}


There are two types of WBAN: implanted and wearable. Implanted WBAN nodes are sensors implanted under the human skin where the electrical properties of the body affect the signal propagations [3]. These implanted devices communicate wirelessly to the external device. Wearable BAN nodes are small chips kept over the skin to allow doctors to get real time data about the patients' problems.

\section{Related Work}

Recent works have proposed energy efficient wireless MAC protocol for communication within the WBAN [2]. In [2] the research demonstrates that there is a possibility of communication with intermittent data sources with very low duty cycles. The authors in [3] Studied the observation and monitoring approaches used in WBAN. The researchers discussed the way of measurement that supports the quality of communications to the application and concluded that it is important to evaluate the margin of error or uncertainty of any collected sign or value during the medical monitoring process. In [4] the performance of a remote patient monitoring system using an OPNET based simulation model WBAN is studied. The results of the study in raise two main design issues. Primarily results show that WBAN should not be directly connected to a main hospital network through the service node. Secondly it is noticed that TCP protocol used in a fixed network does not work well in wireless networks. Therefore this research recommends using a combination of wireless and fixed network links to optimize the transmission time (known as multi-hop network). The work in [5] addresses traffic-aware frequency assignment, which considers different nodes' of traffic volumes when making frequency decisions. The research results show that the traffic-aware channel assignment can significantly improve the network performance. The Previous studies touch on the effect of traffic load or type on the performance of a WBAN. However, to the best of our knowledge, traffic modeling in WBAN is not addressed yet. Thus, this work attempts to close this void by designing a traffic model for WBAN.

\section{Requirement \& Analysis}

The WBAN is required to carry traffic with a wide range of QoS requirements. WBAN traffic can be categorized into normal traffic, on-demand, and emergency. Normal traffic is further classified under three categories which are high, medium and low, depending upon the wake up period. The wake up period depends upon the power supply, i.e., on AC power supply the sensor can wake up all the time. On-demand traffic is used for the purpose of diagnosis and prescription. This is further divided into continuous (in case of surgical events) and discontinuous (when occasional information is required). Emergency traffic is initiated by the nodes when they exceed a predefined threshold and should be accommodated in almost no time. The objective of this research is to study the statistical characteristics of the WBAN traffic and the effect of the mixture of the different types of traffic on the critical and real time applications traffic. We believe that traffic modeling will facilitate the design of efficient scheme to provide for improved QoS guarantees in WBAN. Traffic modeling is a technique to study the statistical characteristics of traffic in an attempted to search for standardized stochastic information. Traffic statistics play a vital role on the performance of network. For example, in case of VoIP (Voice over Internet Protocol) when voice traffic is simply transmitted over IP-based networks, traffic modeling is important in this case for flow control and for network congestion management. Therefore, resource management and QoS guarantees are among the most important tasks in e-health care services. Another example of the importance of traffic modeling is to simulate and reflect real-time functions where traffic modeling is used to adjust each case based on a set of parameters and values assigned for each environment and each case. For example, transmitting X-Ray images has its own bandwidth and performance needs. So traffic modeling simulation permits the adaptation of some parameters to improve the QoS support for transporting XRay traffic. The main objective of this work is to study and deduce a traffic model for WBAN using simulation. Using simulation methods for traffic modeling comprises 4 major steps: Modeling the system with its dynamics, generating and realization traffic, measuring the simulated data and analyzing the output data. Hence, we will simulate three ZigBee network topologies: Mesh, Star, and Tree, using the OPNET module with the same number of network nodes when applicable. We will generate different types of workload that simulates realistic WBAN traffic as much as possible. Over the operative network, we will collect data from the network and analyze the collect data to conclude some modeling parameters. However, before presenting the design and experimentation, we survey current related work to our objective. 


\section{Design and Architecture}

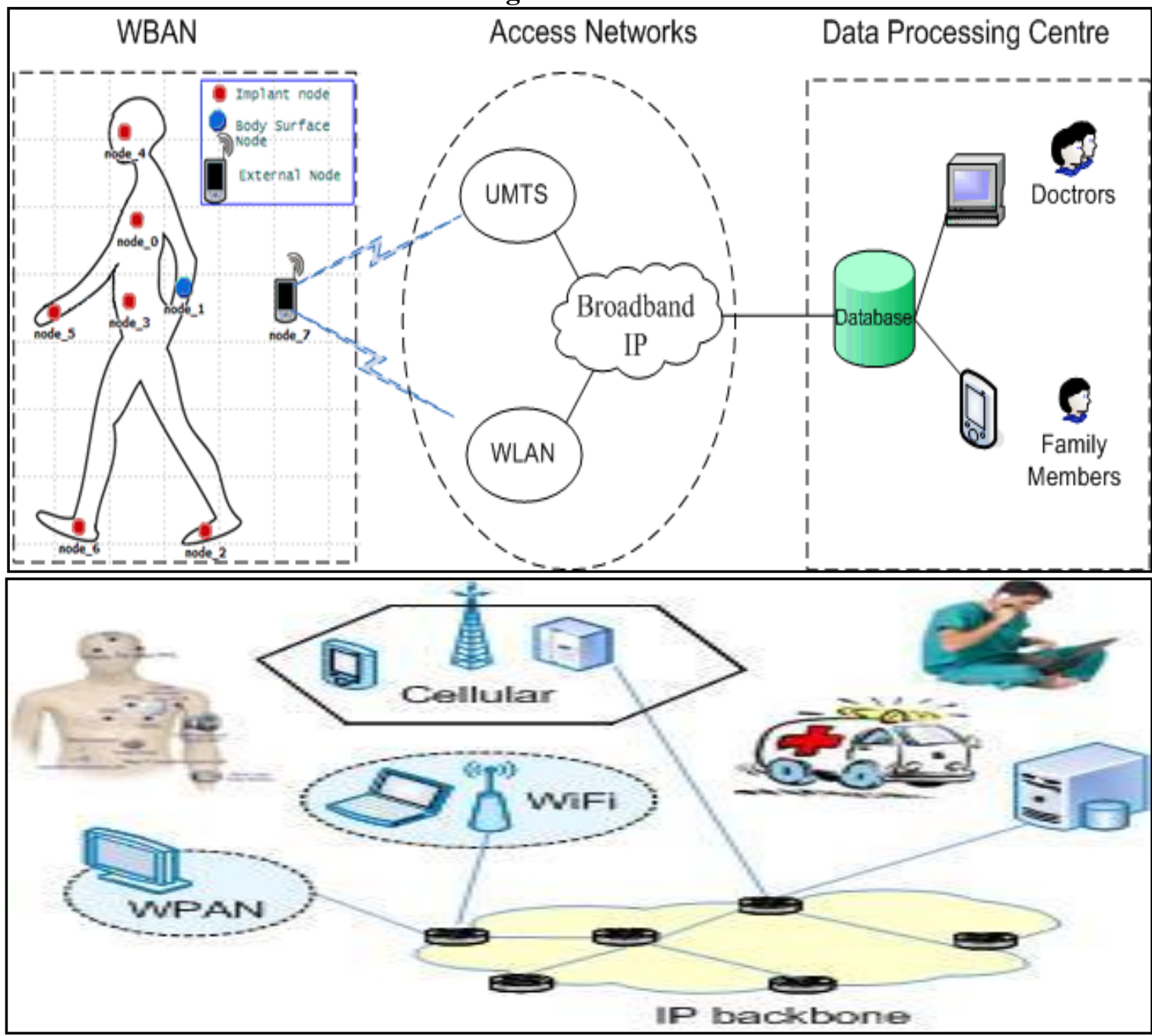

Figure 1: Overview of Traffic Modeling of Wireless Body Area Network

In our project we use the OPNET (Optimized Network Engineering Tool) software which is a powerful communication system simulator developed by OPNET Technologies. We create our network by using the OPNET and we configure three types of topologies: Mesh, Star, and Tree. The Mesh Topology consists of ZigBee coordinator, node routers and end nodes. Mesh topology is considered the most flexible topology of the three because a message can take multiple paths from source to destination. If a particular router fails, then ZigBee's self-healing mechanism (or route discovery) will allow the network to search for an alternate path for the message. The Star Topology consists of the ZigBee coordinator and multiple end nodes, in which the end nodes are directly connected to the ZigBee coordinator in a point-to-multipoint communication mode. This type of topology is attractive because of its simplicity. However, it has a major disadvantage, the ZigBee coordinator is one point of failure because all traffic must travel through the ZigBee coordinator of the star. The last topology is the Tree Topology, in a tree network; a coordinator is the root of the tree. The coordinator can now have either routers or end devices connected to it. For every router connected, more child nodes can connect to the router. Child nodes cannot connect to an end device because they do not have the ability to relay messages. The disadvantage of the tree topology is the failure of a router. If a router fails, all of that router's children are cut off from communicating with the rest of the network.

We ran different types of traffic over the ZigBee network. Constant bit rate traffic simulates voice or file transfer data and variable Bit rate traffic simulates, for example, video traffic. The modeling parameters chosen in our project are: delay and throughput. In addition, we observed the results for the received traffic in each topology 


\section{Implementation and Testing}

We implemented six scenarios in this project: tree mesh and star topology with constant and variable bit rate traffic, the study parameters for the three different topology types were compared with each other. There was one ZigBee coordinator in each topology; therefore each network formed a single Personal Area Networks (PAN). The comparison included end-to-end delay and throughput.

Mesh, Star, and Tree topologies in a ZigBee network were studied. Each network consists of one ZigBee coordinator (ZC). The number and type of the devices were identical when applicable. The Mesh Topology is designed as a single personal area network PAN as shown in Figure 2. We placed one coordinator node, six routers and eleven end devices in the workspace. On each device, we confirmed that the network parameters attribute is set to default mesh network. The Star Topology is designed by placing one coordinator node and eleven end devices in the workspace. On each device, we confirmed that the network parameters attribute was set to default star network as shown in Figure 3. The Tree Topology is created by placing one coordinator node, eight routers and seventeen end devices in the workspace as shown in Figure 4.

Simulated time for all three setups was 1,200 seconds. Every device in the network sent a packet of 1024 bytes to a random destination with the interval of 1 second.

\section{Results}

The focus of the study of the scenarios is mainly on the end-to-end delay, the throughput and the traffic received. The end-to-end delay is shown in Figure 5 shows the end-to-end delay result with constant dtat traffic over the three topologies. We observed that the three topologies have different end-to-end delay in this simulation. The tree topology has a highest end-to-end delay. The mesh topology has the second highest global end-to-end delay and finally, the star topology has the lowest global end-to-end delay. The star topology incurs the lowest delay because the traffic is directly routed to the Zigbee Coordinator (ZC). Mesh has lower delay than tree due to the reliability of routes; the traffic can be routed over more than one route than that provided by tree which is only one route.

Figure 6 shows the end-to-end delay result for the variable data traffic. The Tree topology has a higher end-to-end delay due to the burstness of the traffic. The mesh and star topology show comparable delays because in case of star the end devices are directly connected to the ZC. For the case of mesh, traffic can be always routed over different routes. Hence, the delay is lightly increased. Figure 7 shows the delay histogram of the mesh network and Figure 8 shows the probability density function (pdf) as an example of fitting the end-toend delay into will known distributions. As Figure 7 and 8 shows the pdf cannot be mapped to any standard pdf. It can only be observed as an empirical pdf. Additionally, since the traffic generator of the OPNET simulator is based only on ZigBee traffic, that is the OPNET general traffic generator cannot be used in simulation, the OPNET with its current format cannot be employed for trusted results for traffic modeling. This is also valid for the histogram of traffic received for the mesh topology and the traffic received PDF of the mesh network shown in Figure 9 and Figure 10 consequently.

Figure 11 shows the results for throughput with constant bit rate traffic. The tree topology has the highest throughput. The mesh topology has the second highest throughput. Finally, the star topology has the lowest throughput. This is due to the fact that traffic is carried out by routers, end-nodes and ZigBee coordinator in case of tree and mesh topology. However, in case of star topology, traffic is only routed over the ZC. Figure 12 shows similar throughput result with variable data rate.

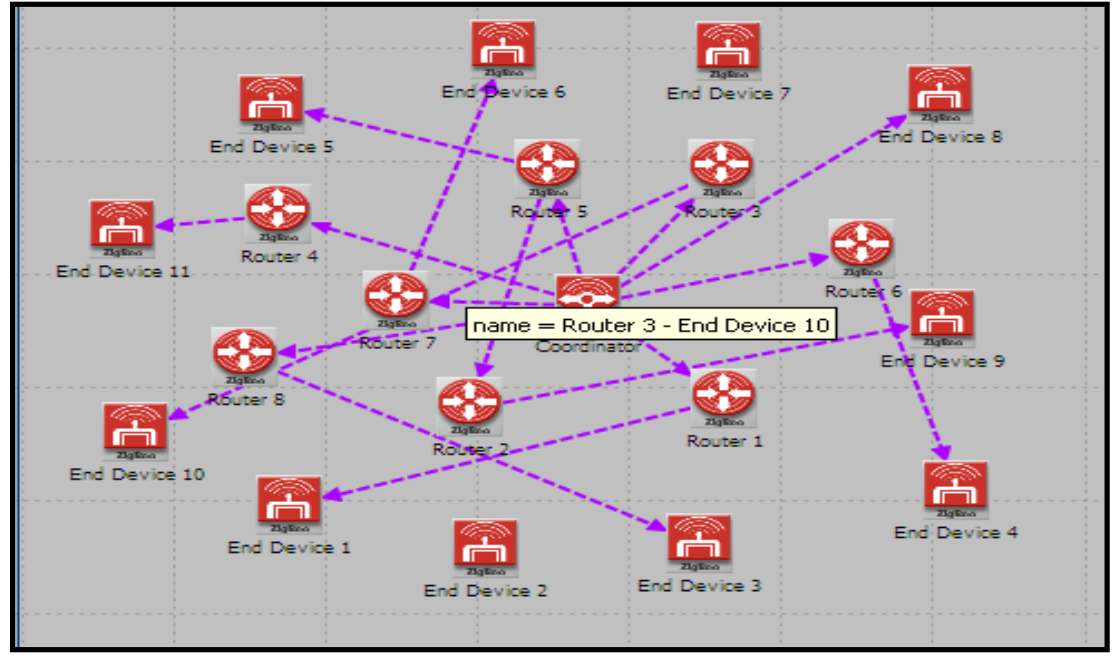

Figure 2: Mesh Topology 


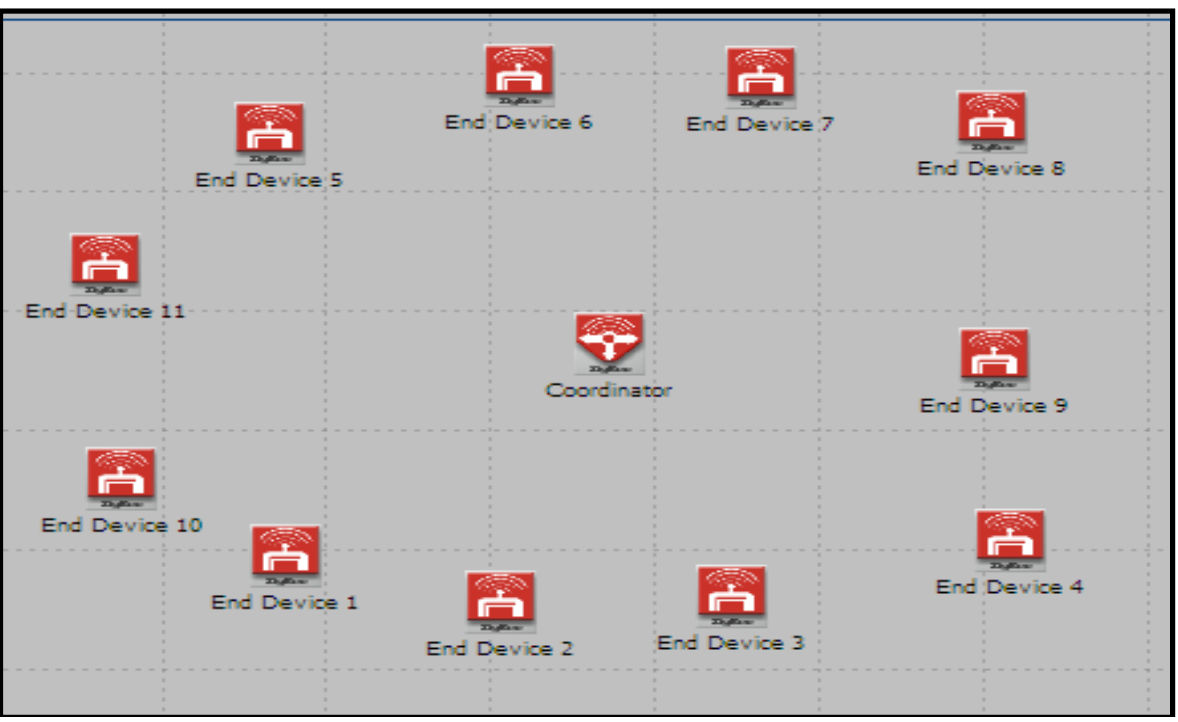

Figure 3: Star Topology

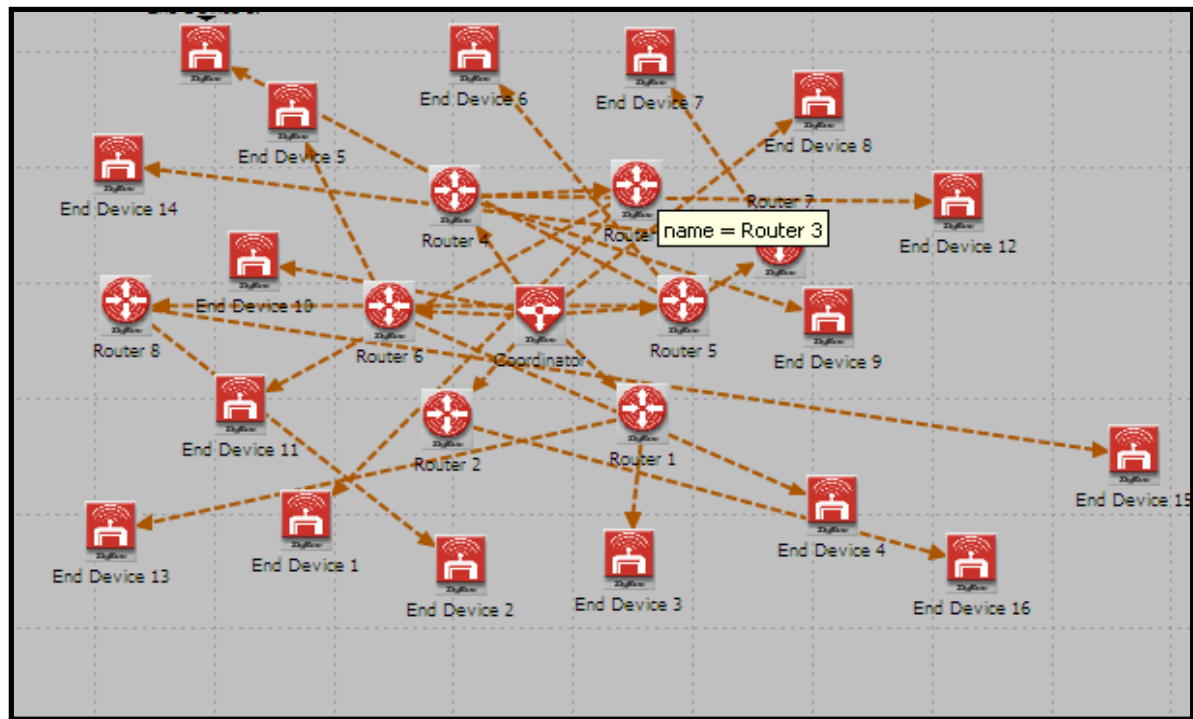

Figure 4: Tree Topology

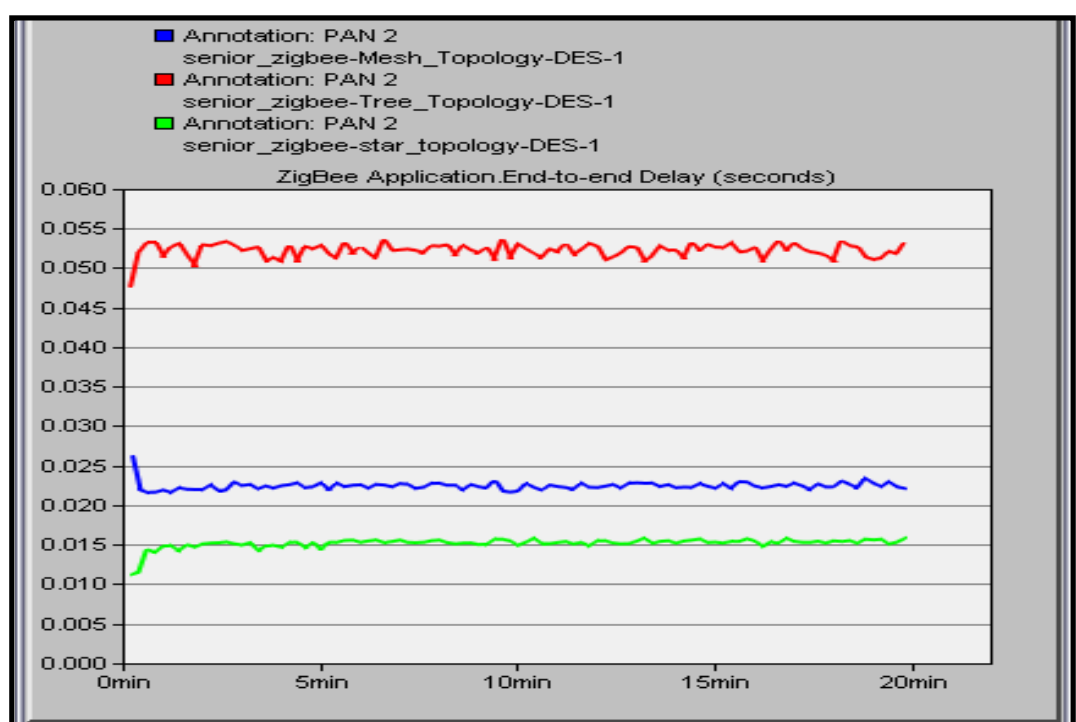

Figure 5: End-to-end delay of the three topologies with constant bit rate 


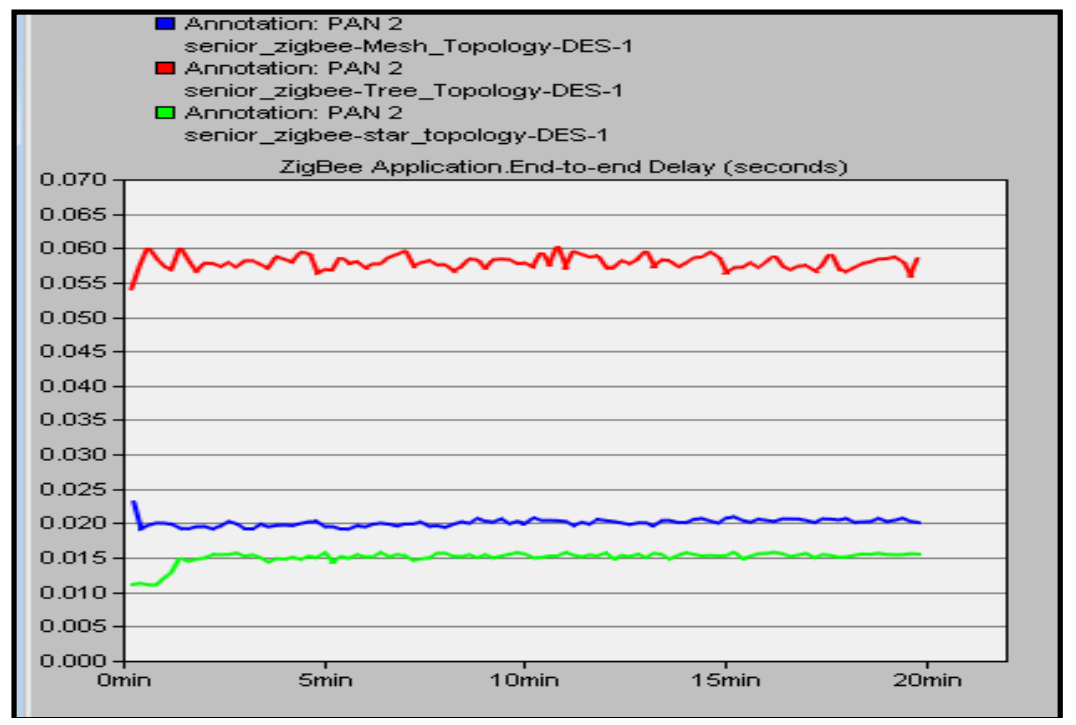

Figure 6: End-to-end delay of the three topologies with variable bit rate

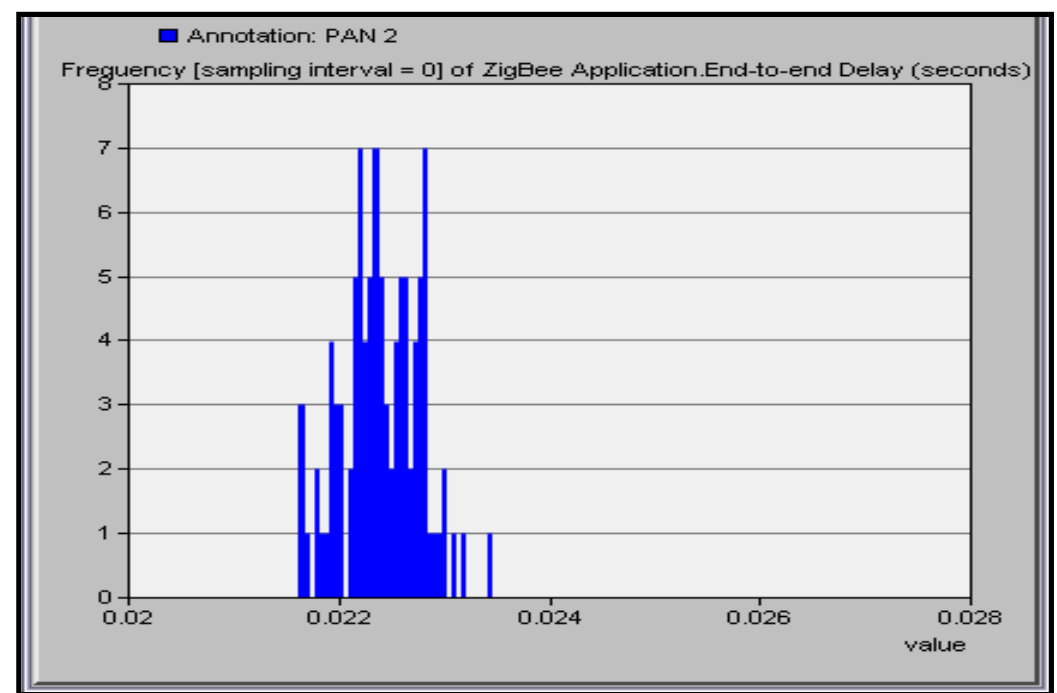

Figure 7: Histogram of end-to-end delay of the mesh network

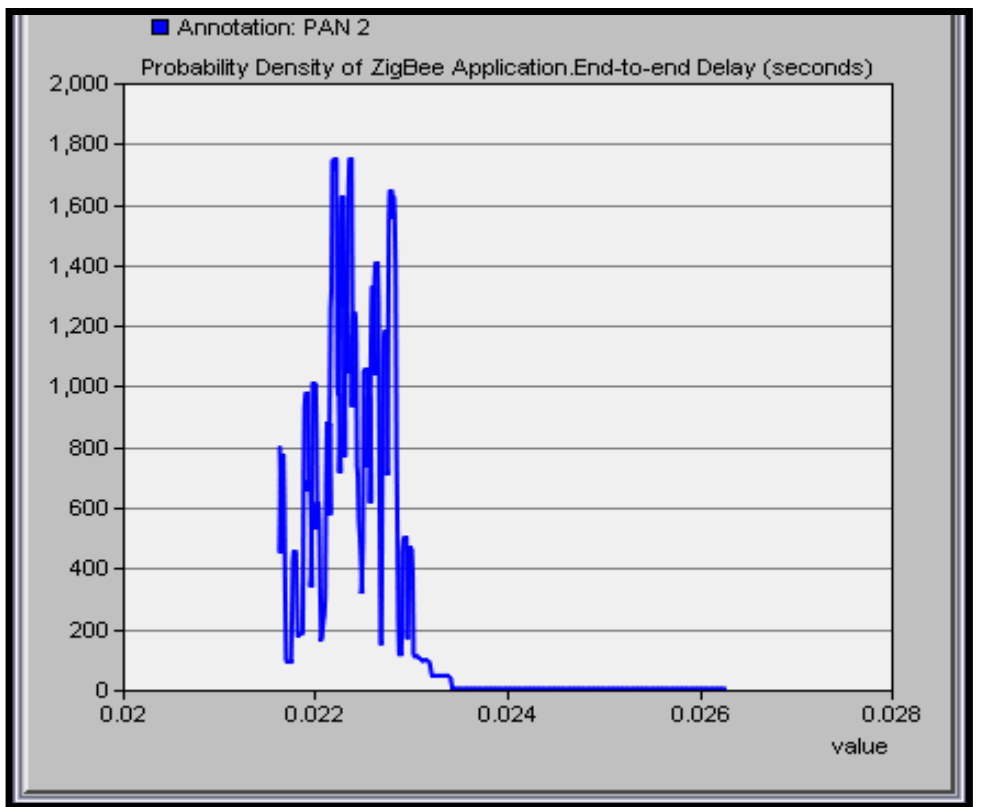

Figure 8: Pdf of end-to-end delay of the mesh network 


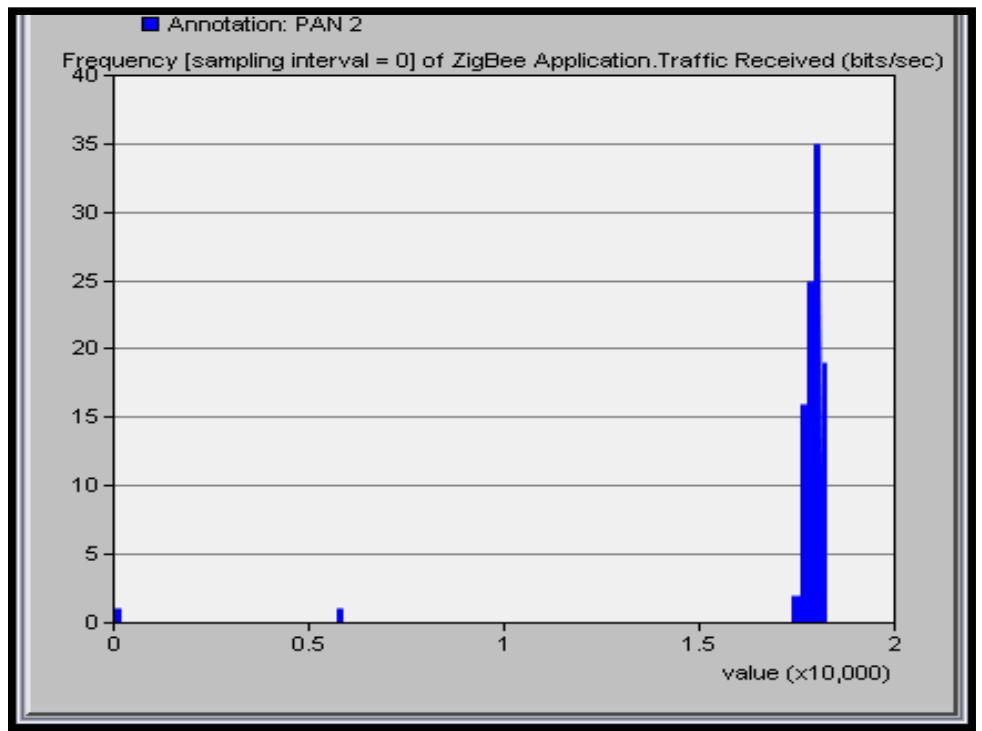

Figure 9: Histogram of the received traffic of the mesh network

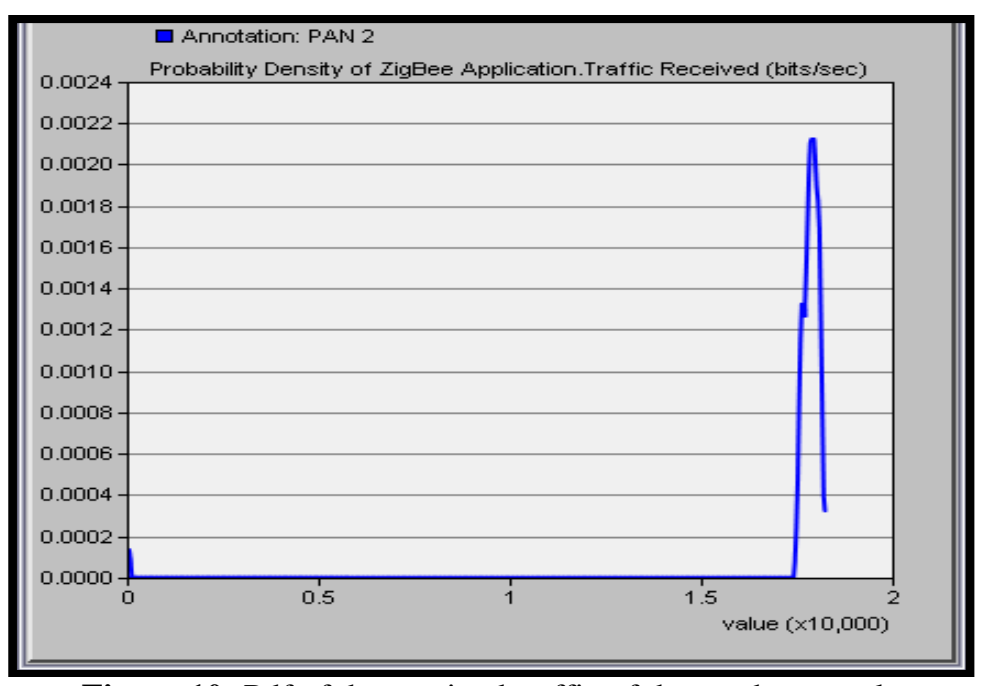

Figure 10: Pdf of the received traffic of the mesh network

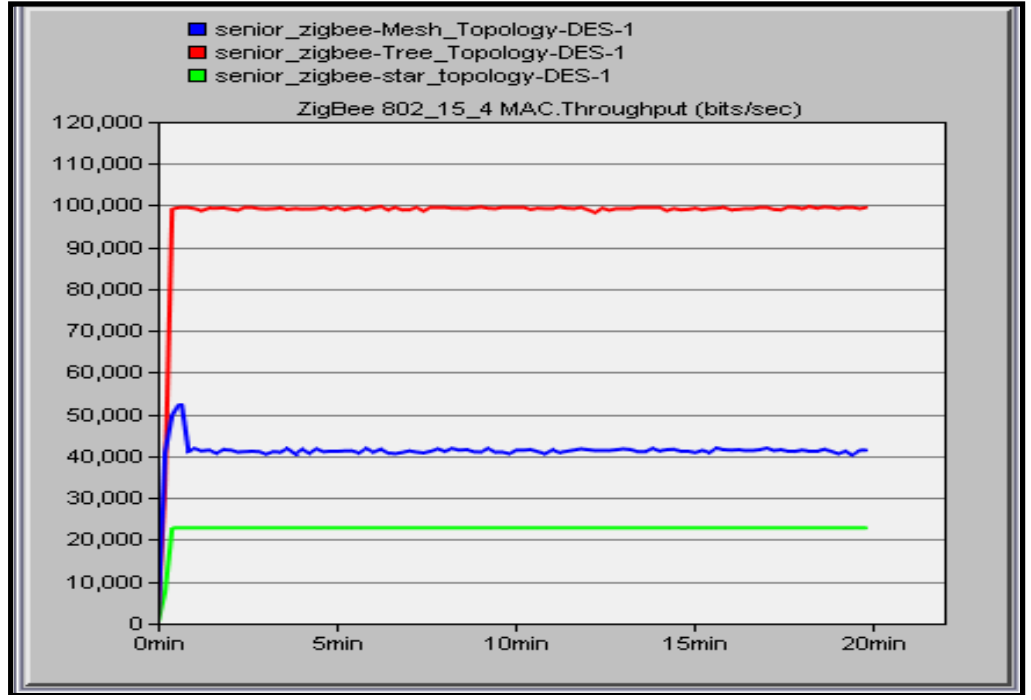

Figure 11: Throughput of the three topologies with constant bit rate 


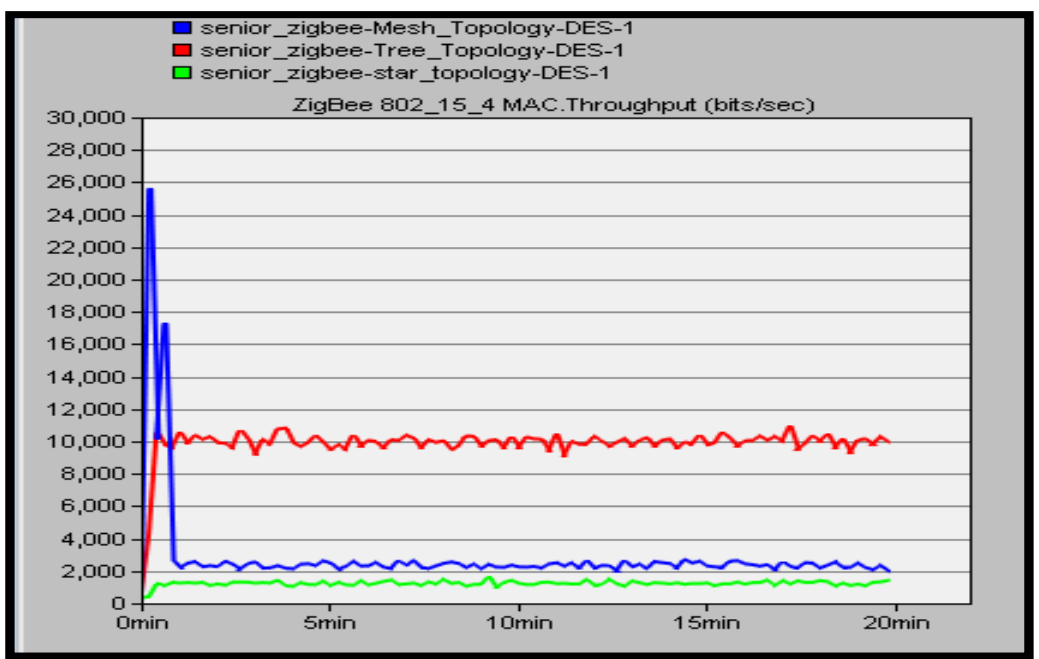

Figure 12: Throughput of the three topologies with variable bit rate

Through this project, we have learned some of the limitations about the ZigBee model in OPNET. The most noticeable limitation of the ZigBee model is: in the traffic model, all ZigBee components of OPNET send and receive ZigBee traffic only. It cannot use the traffic generator of the OPNET itself. The ZigBee OPNET model does not support a contention-free mode to support real time traffic. Hence, providing scheduling algorithms for real time traffic is not supported. Additionally, OPNET does not provide source code for the ZigBee network layer. This layer is intentionally provided without any source code and only object code is provided. Access to the source code is essential to implement some mechanisms for ZigBee. Another major limitation of ZigBee is that it does not provide a gateway entity for connecting the ZigBee coordinator into other networks such as WiMAX or LTE. This stopped us investigating the implementation of a gateway to interconnect the ZigBee network with other networks. We concluded that the current design of the OPNET module does not provide enough tools to design and implement a ZigBee gateway.

\section{Conclusion}

We investigated the traffic behavior in a ZigBee network in three topology modes: tree, mesh and star. We observed that the star topology entails the lowest delay due to the direct communication between the ZC and the end-nodes. We also observed that the traffic statistics cannot be modeled to a standard PDF format due to the fact that the results cannot be matched to a known PDF distribution and most importantly, the ZigBee module of the OPNET simulator does not support the OPNET general traffic generator. Additionally, the OPNET ZigBee module has several limitations but mainly it does not support gateway node functionality of a ZigBee network.

\section{References}

[1]. Latré, B., Moerman, I. \& Dhoedt, B. (2011, April 26). Networking in Wireless Body Area Networks, p.1.

[2]. Marinkovic, S., Spagnol, C. \& Popovici, E. (2010, March 26). Energy-Efficient TDMA-based MAC Protocol for Wireless Body Area Networks

[3]. Orhan, I., Gonga, A. \& Lindh, T. (2010, April 10). An End-to-End Performance Meter for Applications in Wireless Body Sensor Networks

[4]. Khan, J., Yuce, M. \& Karami, F. (2010, March 16). Performance Evaluation of a Wireless Body Area Sensor Network for Remote Patient Monitoring

[5]. Yafeng, W., Keally, M. \& Zhou, G. (2011, February 20). Traffic-Aware Channel Assignment in Wireless Sensor Networks 